\title{
Baboon syndrome
}

\author{
Julia Nowowiejska, Anna Baran, Iwona Flisiak
}

Department of Dermatology and Venereology, Medical University of Bialystok, Bialystok, Poland

Adv Dermatol Allergol 2022; XXXIX (2): 413-415

DOI: https://doi.org/10.5114/ada.2020.98721

'Baboon syndrome' (BS) is a term primarily used to describe a mercury-induced skin eruption after previous sensitization to this chemical element, which was introduced in 1984 by Andersen et al. [1, 2]. This term is related to the clinical picture of confluent erythematous lesions involving buttocks which resemble red buttocks of baboons [3, 4]. Since then, there have been multiple case reports considering this clinical description but taking into account various pathogenesis: drug-induced and non-drug induced (allergenic and non-allergenic). Therefore, a great confusion regarding this term occurred and some researchers have tried to review this syndrome creating a new classification [2].

A 74-year-old man, with the history of arterial hypertension, gout, hepatic cirrhosis and osteoarthritis, was admitted to the Dermatology Department in order to diagnose and treat skin lesions persisting for 5 days. The day the lesions occurred the patient was discharged from the orthopaedic ward where he had undergone left hip cementless endoprosthesoplasty due to osteoarthritis. CORIN Trinity 56 acetabular cup, asymmetrical liner, Minihip 6 stem, 40/-4 head had been implanted. At hospital he had also received multiple medications, e.g. enoxaparin, oxycodone, pseudoephedrine, bupivacaine, tramadol and gentamicin. The first skin lesion, an erythematous patch, appeared on the thigh while the patient was just about to be discharged from the orthopaedic ward. After coming back home an eruption of new erythematouspapular lesions occurred, therefore the patient called an ambulance and he was administered intramuscular antihistamines and intravenous glucocorticoids. On the third day, another flare appeared, therefore the patient presented to the Department of Dermatology. On admission confluent erythematous-infiltrative lesions in the area of buttocks (Figure 1), neck and cleavage in a V-shape were observed as well as symmetrical erythematous-infiltrative and erythematous-papular lesions within the back, thighs, cubital and axillary fossae (Figures 2, 3). The skin lesions were not accompanied by fever or joint pain. Of the laboratory investigations there was normocytic anae- mia and slight C-reactive protein elevation observed with no eosinophilia. During the interview the patient reported two similar episodes in the past: 13 years earlier he was diagnosed with drug rash with eosinophilia and systemic symptoms (DRESS), most probably due to allopurinol, colchicine or nonsteroidal anti-inflammatory drugs. Moreover, 7 years earlier, a very similar reaction occurred, probably due to allopurinol or naproxen as well, therefore he was hospitalized at our Department, but at that time he did not fulfil the criteria of DRESS, so the diagnosis of dermatitis due to unspecified substance taken internally was made. During the current episode at our Department, enoxaparin was stopped, replaced by an oral anticoagulant, hydrocortisone intravenously $100 \mathrm{mg}$ three times a day and topical glucocorticoid ointments were introduced. A spectacular improvement was noted: on the second day of hospitalization, erythema of the cleavage and limbs resolved. We contacted the orthopaedic surgeons who performed the patient's surgery and discussed further proceedings. The patient was scheduled for patch tests after stopping the therapy with glucocorticoids and antihistamines. Basing on the

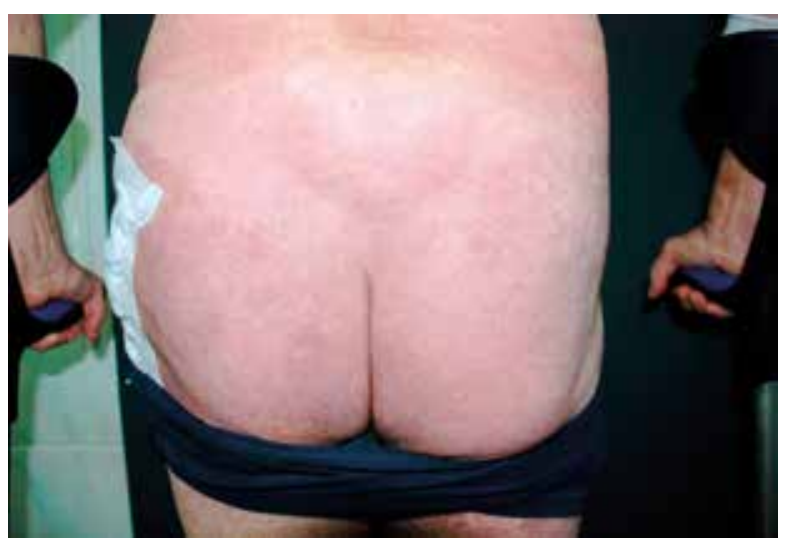

Figure 1. Symmetrical erythematous-infiltrative lesions on both buttocks. Dressing after the hip surgery on the left buttock

Address for correspondence: Julia Nowowiejska MD, Department of Dermatology and Venereology, Medical University of Bialystok, 14 Zurawia St, 15-540 Bialystok, Poland, phone/fax: +85 74095 70, e-mail: julia.nowowiejska94@gmail.com Received: 2.07.2020, accepted: 7.08.2020. 


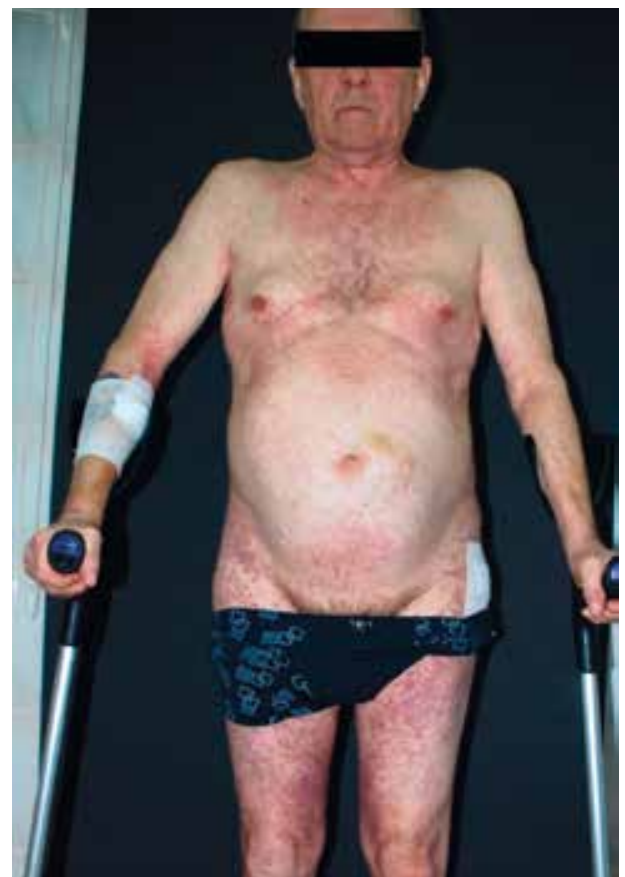

Figure 2. Confluent erythematous V-neck shaped lesions on the neck and cleavage, erythematous-papular lesions over the skin of the thorax and abdomen. Skin lesions also located in the flexural areas - cubital and axillary fossae, as well as lesions in the shape of reverse triangle in the area of the medial thigh surface

patient's medical history and physical examination, as well as laboratory tests, the baboon syndrome was diagnosed. Most probable inducing factors were the medications given during orthopaedic surgery, however it was not possible to identify the specific drug as the ultimate trigger. After administered treatment the skin lesions resolved completely and the patient was discharged home with a recommendation of follow-up in an ambulatory care setting.

Different pathogenic mechanisms can result in the clinical picture of the baboon syndrome but there are three main suggested [2]. First, the classical BS occurs due to parenteral allergen intake after primary contact sensitization to the same substance, excluding drugs $[2,5]$. The second type of BS is contact-allergenic druginduced BS and the third is non-contact allergenic drug induced BS called symmetrical drug-related intertriginous and flexural exanthema (SDRIFE) [2]. Nevertheless, the diagnosis is based on clinical features: (1) well-demarcated erythema involving the gluteal or perianal area and/or V-shaped erythema in the inguinal and perigenital area; (2) the involvement of at least one intertriginous or flexural area; (3) symmetry of the affected areas; and (4) the absence of systemic symptoms [2]. The reason for such flexural localization of lesions is not certain, but it is suspected to be due to sweating and local occlusion [4]. Our patient has met all of the listed criteria, although

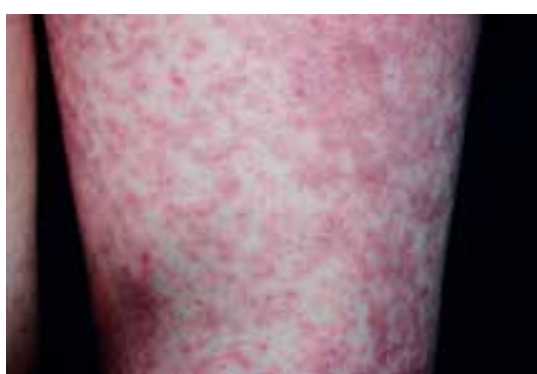

Figure 3. Close-up view of the skin lesions. Erythematouspapular lesions on the thigh

the specific drug causing them all has not been identified as he received such broad treatment. BS can be caused by either topically or systemically applied substances. Among the systemic offending agents most commonly responsible are: $\beta$-lactam antibiotics, especially amoxicillin, mercury, nickel, pseudoephedrine, contrast media containing barium, allopurinol, oxycodone, naproxen, heparin, oral neomycin, oral glucocorticoids and many more, whereas our patient received even four of them [2]. The probable time interval between drug administration and occurrence of skin lesions is usually from a couple of hours to 2 days [6]. Other possible triggers for clinical manifestation of BS may be infections, bacterial (especially streptococcal) or viral [2]. There is no specific test confirming the diagnosis - it is based on the clinical picture and exclusion of other potential causes, although the cause and effect relationship with a medication can be confirmed in the controlled drug provocation test [6]. Usually, there are no general symptoms, and laboratory data or histopathological examination are not useful [2]. Skin lesions are often self-limiting, although intravenous glucocorticoids, which shorten the duration of the disease, can be administered [6]. In the described case, after administration of intravenous hydrocortisone in lowering doses starting with $100 \mathrm{mg}$ three times a day, as well as intramuscular and oral antihistamines and topical hydrocortisone ointment, the lesions improved quickly and at the end of hospitalization, 8 days altogether, resolved completely.

\section{Conflict o interest}

The authors declare no conflict of interest.

\section{References}

1. Andersen KE, Hjorth N, Menne T. The baboon syndrome systemically-induced contact dermatitis. Contact Dermatitis 1984; 16: 97-100.

2. Miyahara A, Kawashima H, Okubo Y, Hoshika A. A new proposal for a clinical-oriented subclassification of baboon syndrome and a review of baboon syndrome. Asian Pac J Allergy Immunol 2011; 29: 150-60.

3. Blackmur JP, Lammy S, Baring DE. Baboon syndrome: an unusual complication arising from antibiotic treatment of 
tonsillitis and review of the literature. BMJ Case Rep 2013; 2013: bcr2013201977.

4. Jankowska-Konsur A, Kolodziej T, Szepietowski J, et al. The baboon syndrome - report of two first cases in Poland. Contact Dermatitis 2005; 52: 289-90.

5. Omulecki A, Olejniczak-Staruch I, Torzecka J. Baboon syndrome. Symmetrical drug-related intertriginous and flexural exanthema. Dermatol Rev 2013; 100: 265-8.

6. Arnold DF, Sargur R, Nakonechna A. Drug hypersensitivity testing: baboon syndrome precipitated by amoxicillin challenge. Allergy Asthma Immunol 2020; 124: 104-6. 